\title{
Fetal alcohol exposure impairs hedgehog cholesterol modification and signaling
}

\author{
Yin-Xiong Li ${ }^{1,2,3}$, Hai-Tao Yang ${ }^{1,5}$, Marzena Zdanowicz ${ }^{1,5}$, Jason K Sicklick ${ }^{1,4,5}$, Yi Qi ${ }^{1}$, Terese J Camp ${ }^{1}$ and \\ Anna Mae Diehl ${ }^{1}$
}

Consumption of alcohol by pregnant women can cause fetal alcohol spectrum defects (FASD), a congenital disease, which is characterized by an array of developmental defects that include neurological, craniofacial, cardiac, and limb malformations, as well as generalized growth retardation. FASD remains a significant clinical challenge and an important social problem. Although there has been great progress in delineating the mechanisms contributing to alcohol-induced birth defects, gaps in our knowledge still remain; for instance, why does alcohol preferentially induce a spectrum of defects in specific organs and why is the spectrum of defects reproducible and predictable. In this study, we show that exposure of zebrafish embryos to low levels of alcohol during gastrulation blocks covalent modification of Sonic hedgehog by cholesterol. This leads to impaired Hh signal transduction and results in a dose-dependent spectrum of permanent developmental defects that closely resemble FASD. Furthermore, supplementing alcohol-exposed embryos with cholesterol rescues the loss of Shh signal transduction, and prevents embryos from developing FASD-like morphologic defects. Overall, we have shown that a simple post-translational modification defect in a key morphogen may contribute to an environmentally induced complex congenital syndrome. This insight into FASD pathogenesis may suggest novel strategies for preventing these common congenital defects.

Laboratory Investigation (2007) 87, 231-240. doi:10.1038/labinvest.3700516; published online 22 January 2007

KEYWORDS: fetal alcohol syndrome; fetal alcohol spectrum defects; hedgehog; cholesterol; post-translational modification; signal transduction

Post-translational protein modification plays an essential role in facilitating signal transduction regulation of gene expression. Protein modification by phosphorylation, acetylation, or methylation helps control the proper timing and sequence of events during embryogenesis; therefore, it is not surprising that defective modifications of these proteins can be important causes in the development of many types of congenital diseases. Accumulating evidence illustrates the importance of post-translational lipid modifications for regulating protein function. One example is the cholesterol and palmitoyl modification of Sonic hedgehog (Shh), which guides this protein's biogenesis, cellular trafficking, and functionality. ${ }^{1}$

Shh is a highly conserved fetal morphogen that plays a central role in cell proliferation, differentiation, and embryonic patterning by activating the Hedgehog $(\mathrm{Hh})$ signal pathway. ${ }^{2,3}$ The $45 \mathrm{kDa}$ Shh precursor protein undergoes modification by auto-processing, followed by covalent linkage of cholesterol to the N-terminal proteolytic product. ${ }^{4}$ This mature, cholesterol-modified protein $(19 \mathrm{kDa})$ can be transported to the cell membrane for secretion. ${ }^{5}$ Once secreted, the cholesterol-modified Shh ligand can initiate signal transduction by binding to its receptor, Patched (Ptc). Upon binding, Ptc relieves the inhibition of the signal transducer, Smoothened (Smo), ${ }^{6}$ which then activates Gli transcription factors by uncoupling them from the negative regulator, Suppressor of Fused. ${ }^{7}$ Gli is subsequently translocated to the nucleus and regulate expression of target genes including Ptc, ${ }^{8}$ Gli1 $^{9}$ itself and Nkx2.2. ${ }^{10}$

During embryogenesis, Shh is expressed specifically in Hensen's node, the floor plate of the neural tube, the cardiac mesenchyme, the early gut endoderm, the posterior portion of the limb buds, and throughout the notochord. As it is a

\footnotetext{
${ }^{1}$ Division of Gastroenterology, Department of Medicine, Duke University Medical Center, Durham, NC, USA; ${ }^{2}$ Department of Cell Biology, Duke University Medical Center, Durham, NC, USA; ${ }^{3}$ Department of Pediatrics, Duke University Medical Center, Durham, NC, USA and ${ }^{4}$ Department of Surgery, Johns Hopkins University School of Medicine, Baltimore, MD, USA

Correspondence: Dr Y-X Li, MD, PhD, Department of Medicine, Duke University Medical Center, Snyderman-GSRB I, Suite 1073, 595 LaSalle Street, Box 3256, Durham, NC 27710, USA. E-mail: yinxiong.li@duke.edu

${ }^{5}$ These authors contributed equally to this work.

Received 9 October 2006; revised and accepted 12 November 2006
} 
morphogen, Shh also affects the development of tissues that are distal to where it is produced. Shh is apparently a key inductive signal for patterning of the ventral neural tube $e^{11,12}$ the anterior-posterior limb axis, ${ }^{13}$ and the ventral somites. ${ }^{14}$ In humans, one severe phenotype caused by mutations in Shh, or other components of Hh signaling, is holoprosencephaly (HPE), ${ }^{15}$ a disorder in which the fetal prosencephalon (forebrain) fails to divide to allow formation of bilateral cerebral hemispheres. HPE is also one of the extreme manifestations of severe fetal alcohol spectrum defects (FASD) in human embryos and in animal models of FASD. ${ }^{15-20}$ Similarly, production of Shh in the floor plate of the neural tube regulates the development of neural components in the overlying basal plate, including progenitors of motor neurons. ${ }^{12,21}$ FASD patients display delayed motor development and impaired fine- and gross-motor skills. ${ }^{22}$ Varying degrees of motor retardation have been observed in up to $89 \%$ of humans having FASD. ${ }^{23-25}$ Indeed, the diagnostic criteria for FASD include impaired fine motor skills. ${ }^{26}$ Shh also has a proven role in neural crest morphogenesis, ${ }^{27}$ and FASD frequently includes defects in neural crest-derived structures. Clearly, there is significant overlap between the tissues affected by alcohol exposure, and those tissues that depend on Shh signaling for proper development.

Post-translational modification of Shh by cholesterol ${ }^{4}$ is a tightly regulated process that is necessary for the transportation and establishment of concentration gradients of the mature Shh ligand in developing embryos. ${ }^{28}$ Sterol- and fatty acid-modified Shh proteins form soluble multimers that are packaged in micelles for long range transport. ${ }^{29}$ Recent work has demonstrated that the activity and function of Shh protein varies significantly, depending upon the presence or absence of these modifications. ${ }^{5}$ The roles played by cholesterol-modified Shh ligand in many facets of embryogenesis may account for some of the teratogenic effects of perturbed cholesterol biosynthesis in animal development. ${ }^{30}$ Similar congenital defects occur in offspring of women who drink alcohol during pregnancy.

The teratogenic consequences of fetal exposure to alcohol are highly variable and include a spectrum of morphologic defects known as FASD. ${ }^{31}$ Phenotypic abnormalities of FASD include neurological, craniofacial, cardiac, and limb malformations, as well as generalized growth deficits and mental retardation. ${ }^{32}$ The mechanisms proposed to underlie the spectrum of birth defects caused by fetal alcohol exposure include: apoptosis, ${ }^{33}$ cell adhesion defects, ${ }^{34}$ accumulation of free radicals, ${ }^{35}$ dysregulation of growth factors, ${ }^{36}$ and altered retinoic acid biosynthesis. ${ }^{37}$ Some simple and essential questions have not been well explained by these hypotheses, for instance, how one or, at most, a few social drinks, cause fetal defects, why alcohol preferentially induces defects targeting some organs and tissues and not others, or why the pattern of defects seen in FASD is predictable and reproducible.

Alcohol can also impair prechordal plate migration ${ }^{38}$ and disrupt the formation and function of Spemann's Organi- zer, ${ }^{39}$ a signaling center in gastrulating embryos that controls the patterning of the germ layers; the specific mechanisms that regulate axis pattern formation require highly evolutionarily conserved genetic pathways involving transcription regulatory circuitry and signal transduction pathways. ${ }^{40} \mathrm{Shh}$ containing vesicles contained within the organizer initiate a signal transduction pathway that plays a key role in embryonic patterning during development. ${ }^{41}$ Therefore, genetic or environmental factors that inhibit Shh signaling during gastrulation can disrupt proper patterning of the embryo. Interestingly, embryos that are exposed to alcohol during gastrulation ${ }^{38}$ have defects that are similar to those found in embryos that have defects in $\mathrm{Hh}$ signal transduction. ${ }^{42} \mathrm{~A}$ similar phenotype develops in embryos with a genetic defect in sterol homeostasis, for example, Smith-Lemli-Opitz Syndrome $^{43}$ or that are exposed to cholesterol lowering agents. ${ }^{44,45}$ These observations suggest that FASD may result from alcohol-dependent inhibition of cholesterol modification of Shh.

Many genetic disorders can result in abnormal regulation of cholesterol biosynthesis, storage, and trafficking. However, ethanol ingestion may be far more common mechanism for disrupting cholesterol homeostasis. Ethanol causes an inhibition of HMG-CoA reductase activity, which results in decreased free cholesterol in the cells, and reduction in circulating cholesterol levels. ${ }^{46-48}$ Acute ethanol exposure in perfused rat liver results in depletion of cholesterol in both liver homogenate and microsomes. ${ }^{49}$ Ethanol specifically inhibits hepatic ACAT activity, which can lead to decreased cholesterol esters for transport in LDLs. ${ }^{50}$ Thus, evidence from embryology, toxicology, and molecular biology indicates that a teratogenic mechanism underlying FASD links alcohol, cholesterol homeostasis, Shh signaling and cholesterol modification of functional Shh.

Several animal models have been to study FASD. The zebrafish model offers many advantages compared to insect and rodent models for alcohol and development studies: zebrafish are small in size, they have a large number of progeny, and they have rapid embryogenesis. This model has already been widely used in studies of developmental biology, genetics, gene function, signal transduction and high throughput drug screening. All of these characteristics make it an ideal model to delineate the molecular basis of the alcohol-induced birth defects.

We have shown that transient alcohol exposure during early development of zebrafish embryos causes dose-related inhibition of Hh signal transduction and produces a spectrum of permanent FASD-like defects. Alcohol-induced inhibition of Hh pathway activity parallels alcohol disruption of cholesterol homeostasis and decreased cholesterol-modification of the Shh ligand. Supplementing the alcohol-exposed embryos with cholesterol rescues the loss of Shh signal transduction, and prevents embryos from developing FASDlike morphologic defects. 


\section{MATERIALS AND METHODS}

\section{Alcohol, Cyclopamine, and AY-9944 Treatment}

Embryonic alcohol exposures were adapted from a previous report. ${ }^{38}$ Embryos with chorions were exposed to six different concentrations of alcohol (eg, $0,0.25,0.5,1.0,1.5$, and $2.0 \%(\mathrm{v} / \mathrm{v}))$ in embryo medium. Embryos in sealed Petri dishes were exposed to alcohol for $6 \mathrm{~h}$ beginning at the dome stage (ie, 4.25 hours post-fertilization (hpf) or $30 \%$ epiboly stage) and were incubated at $28.5^{\circ} \mathrm{C}$. Immediately following alcohol exposure, embryos were harvested for analysis of Hh pathway activity, cholesterol content, or tissue alcohol concentration. The remaining embryos were washed and incubated in alcohol-free medium. Embryos were then harvested at 1, 2, or 4 days post-fertilization for survival and phenotypic analyses. Cyclopamine (11-deoxojervine) is naturally-occurring chemical that inhibits the Hh signaling pathway by functioning as an antagonist of Smo protein. AY9944, trans-1, 4 bis-(2-dichlorobenzylaminomethyl) cyclohexane dihydrochloride blocks cholesterol synthesis through inhibition of 7-dehydrocholesterol reductase. AY$9944(7.5 \mu \mathrm{M}$, Sigma-Aldrich) and cyclopamine $(10.0 \mu \mathrm{M}$, Calbiochem) were administered in the same manner as alcohol. Following treatment, embryos were washed and incubated in normal medium for up to 4 days postfertilization.

\section{Alcian Blue Staining and Immunohistochemistry}

Staining for skeletal structures was performed as previously described. ${ }^{51}$ Immunohistochemistry is carried out with following primary antibodies (Hybridoma Bank, 1:10): MF20 to stain myocardium and facial muscles, S46 to identify ventricular myocardium, and F59 to identify slow muscle progenitors in the somites. The secondary antibodies were Alexa 568 -conjugated goat anti-mouse $\operatorname{IgG}_{2 \beta}$ and Alexa 488-conjugated goat anti-mouse $\operatorname{IgG}_{2 \alpha}$ (1:400, Molecular Probes). The embryos were mounted and imaged.

\section{Measurement of Cholesterol Content}

Following alcohol exposure, lipids were extracted from embryos $(n \geqslant 14)$ with chloroform-methanol $(2: 1)$ in duplicate. Cholesterol content was then measured by the Amplex Red Cholesterol Assay Kit (Invitrogen).

\section{Measurement of Tissue Alcohol Concentration}

Following alcohol exposure, embryos $(n=38)$ were pooled from each treatment group in triplicate. An Alcohol Test Kit (Diagnostic Chemicals Limited) was used to determine the tissue alcohol concentrations in treated embryos.

\section{RT-PCR and Real-Time Quantitative Analysis}

Total RNA was extracted from embryos $(n=10)$ with RNeasy kits (Qiagen). RT-PCR were performed using with primers (Supplementary Table 1) as described previously. ${ }^{52}$

\section{Immunoblot}

Total cellular protein was isolated as described previously ${ }^{51}$ and cell membrane proteins were isolated using the MemPER(r) Eukaryotic Membrane Protein Extraction Reagent Kit (Pierce Biotechnology). Proteins $(40 \mu \mathrm{g})$ in Laemmli buffer were then separated by $12 \%$ Tris-HCI SDS-polyacrylamide gel electrophoresis and transferred to a PVDF membrane. Membranes were blocked, washed, and exposed to primary antibodies (Santa Cruz Biotechnology) against Shh (N-19, Catalog number: sc-1194; dilution: 1:2500) and $\beta$-actin (1:1000). Signals were detected by Anti-goat HRP antibody (1:10 000, Amersham).

\section{Gli-Luciferase Reporter Assay}

The Gli-luciferase reporter assay was performed in replicate experiments of pooled embryos $(n=15)$. Briefly, zebrafish embryos at the 1-2 cell stage were microinjected with $0.5 \mathrm{nl}$ of Gli-BS-Firefly luciferase plasmid $\left(60 \mathrm{ng} \mathrm{nl}^{-1}\right)$ and Renilla luciferase plasmid ( $60 \mathrm{ng} \mathrm{nl}^{-1}$, pRL-TK, Promega). Reporter activity was determined by using the Dual-Luciferase Reporter Assay System (Promega). Activity of the Firefly luciferase reporter was normalized to the activity of a Renilla luciferase internal control for transfection efficiency.

\section{Cholesterol Microinjection}

Embryos were microinjected at 1-2 cell stage with $0.2 \mathrm{nl}$ of $5 \mu \mathrm{g} \mu \mathrm{l}^{-1}$ (10 pg) cholesterol (BioVision Inc.) with or without the two plasmids for the Gli-luciferase reporter assay. Embryos were allowed to develop and were then treated with alcohol as previously described. At $48 \mathrm{hpf}$ embryos were analyzed.

\section{RESULTS}

\section{Alcohol Exerts Teratogenic Effects in a Dose- and Stage-Specific Manner}

We have chosen the zebrafish model to evaluate our hypothesis because it permits exposure to precise concentrations of alcohol during well-defined developmental time frames. We exposed zebrafish embryos to a range of alcohol concentrations $(0,0.25,0.5,1.0,1.5$, and $2.0 \% \mathrm{v} / \mathrm{v}$ in embryo medium at two different time windows during development. The first exposure window occurs from 1 to 2 cell stages to $3 \mathrm{hpf}$, and the second exposure window occurs between 4.25 and $10.25 \mathrm{hpf}$ during the late blastula stage and the gastrula stage. Exposure to alcohol during the first exposure window is almost uniformly fatal. Fewer than $40 \%$ of the 897 embryos from this time frame that were treated with the lowest alcohol concentration $(0.25 \%)$ survived to $48 \mathrm{hpf}$.

Embryos exposed to alcohol during the second exposure window had much better survival rates than those exposed during the zygote stage to the same levels of alcohol. During the late blastula-gastrula stage, survival of the exposed embryos was also dose-dependent. For example, $<10 \%$ of 202 embryos exposed to $2 \%$ alcohol during this time frame were alive at $48 \mathrm{hpf}$, compared to a survival rate of over $90 \%$ for 
the 897 embryos exposed to $0.25 \%$ alcohol for $6 \mathrm{~h}$ during the same developmental time frame. We also performed more detailed analysis at $48 \mathrm{hpf}$ by scoring alcohol effects in three categories: (a) dead, (b) alive with abnormal phenotype, or (c) alive without abnormal phenotype. As shown in Figure la, during the late blastula-gastrula stage, the phenotype at $48 \mathrm{hpf}$ depended upon the dose of alcohol that embryos were exposed to. For example, $84 \%$ of the embryos exposed to $2 \%$ alcohol during the second exposure window survived through $48 \mathrm{hpf}$ and exhibited abnormal morphology (see Figure 2 for illustrations of representative defects), while only $2.6 \%$ of the living embryos were phenotypically normal. This level of exposure was lethal for the remaining $13 \%$ of embryos. In contrast, $18 \%$ of the embryos exposed to $0.25 \%$ alcohol during the second exposure window were alive and had minimal abnormalities at $48 \mathrm{hpf}$; the majority (72.3\%) were alive and had normal phenotypes and $<10 \%$ failed to survive to the $48 \mathrm{hpf}$ time point. The frequency of these alcohol-induced phenotypes has been characterized in Table 1.

To assure that the alcohol-induced morphologic defects seen in our model do not merely reflect exposure to supraphysiologic concentrations of alcohol, we measured the alcohol concentrations in fetal tissue following alcohol exposures. Tissue alcohol concentrations in zebrafish embryos correspond to the exposed alcohol concentration in embryo medium $0.25-2.0 \%$ range from $0.71-7.4 \mathrm{mM}$ or $0.003-0.034 \mathrm{~g} \mathrm{dl}^{-1}$ (Figure 1b). These alcohol concentrations

a
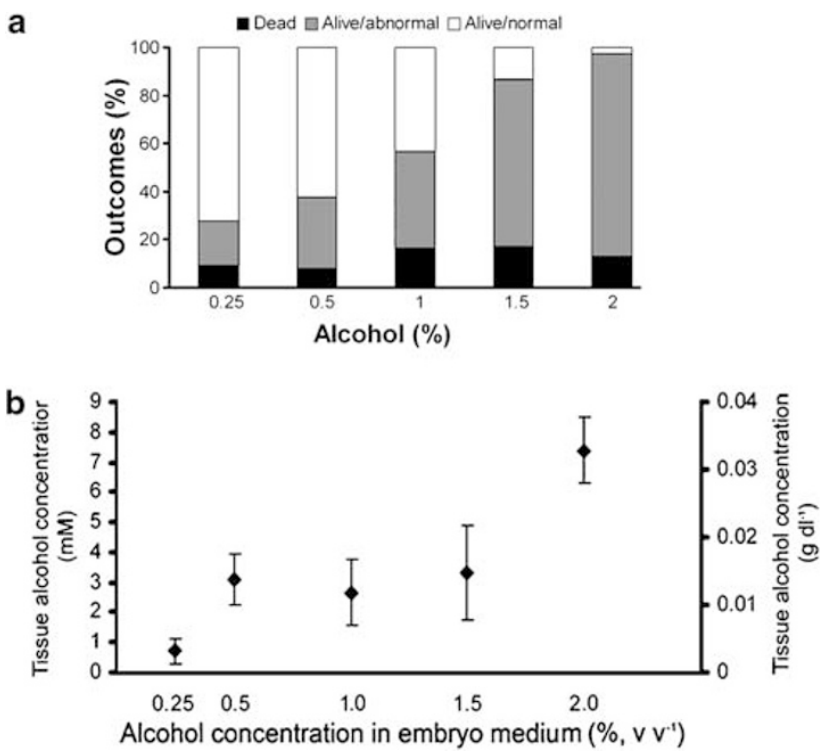

Figure 1 (a) Dose-dependent effects of alcohol exposure on the survival and phenotype of embryos. Embryos $(n \geq 64)$ were exposed to increasing concentrations of alcohol and evaluated at $48 \mathrm{hpf}$. Embryos were scored as alive/normal, alive/abnormal, or dead. These three categories are expressed as a percentage of the total number of embryos in each cohort. (b) Tissue alcohol concentrations in zebrafish are related to the level of alcohol exposure. In general, the alcohol concentrations were in the range of $0.71-7.4 \mathrm{mM}$ or $0.003-0.034 \mathrm{~g} \mathrm{dl}^{-1}$. Error bars indicate 1 s.e.m. of three experiments ( $n=38$ fish per group). can be achieved in the blood of a human being by consumption of one or, at most, a few social drinks.

\section{Morphological Defects Induced by Fetal Alcohol Exposure Recapitulate the Development Abnormities with Hh Signaling Defects}

As others have reported, ${ }^{38}$ we found that alcohol-induced defects in zebrafish embryos recapitulated those that occurred in other species following fetal alcohol exposure. In a dose-dependent fashion, transient alcohol exposure for $6 \mathrm{~h}$ during gastrulation resulted in subsequent permanent phenotypic abnormalities while only a modest increase in the rate of embryo mortality was observed (Figure 1a). By $48 \mathrm{hpf}$, a cumulative cranial-to-caudal phenotype was evident in embryos that were exposed to alcohol transiently during gastrulation (Figure 2). These embryos were growth retarded (Figure 2a), and exhibited a dose-dependent spectrum of phenotypes that included neurological, craniofacial, cardiac, and body axis defects. Embryos exposed to the highest alcohol concentrations had overt HPE, cyclopia (complete or partial), pericardial edema, U-shaped somites and severely foreshortened tails (Figure 2a and b). Comparisons performed between alcohol-treated embryos and embryos transiently treated with cyclopamine, a specific inhibitor of Hh signaling, or AY-9944, an inhibitor of cholesterol biosynthesis and transportation, during gastrulation revealed that embryos from all three groups had HPE and partial cyclopia, as well as underdevelopment of the craniofacial bones and muscles, and failure of the heart tube to loop (Figure 2c). These observations support a role for defective Shh signaling in the pathogenesis of FASD and are consistent with the possibility that alcohol inhibits Shh activity by interfering with cholesterol modification of the cleaved $19 \mathrm{kDa}$ protein.

\section{Alcohol Disrupts Hh Pathway Activity without Significant Change of Shh Protein Expression, but Does Decrease Levels of Cholesterol-Modified Shh}

To investigate this further, Shh signaling activity was directly measured by examining developing embryos that were microinjected with a Shh-responsive, Gli-BS luciferase reporter construct at the 1-2 cell stage. ${ }^{53}$ Following alcohol exposure, both luciferase activity (Figure $3 \mathrm{a}$ ) and the expression of Shh-regulated genes, such as Ptc, Gli1, and Nkx2.2 (Figure 3b), decreased in a dose-related fashion. Real-time quantitative PCR analysis confirmed that there is a threshold for alcohol-induced inhibition of the expression of Shh target genes. While exposure to a very low alcohol concentration $(0.25 \%)$ caused no significant change in the expression of Ptc or Gli1, Nkx2.2 expression decreased by about $50 \%$ under these conditions. In response to a $0.5 \%$ alcohol treatment, expression of these three Shh target genes decreased by from 1.3- to 1.9-fold, and alcohol concentrations ranging from 1.0 to $2.0 \%$ caused expression of those genes to decrease from 5- to 17-fold (Figure 3c). In contrast to the results obtained 
a

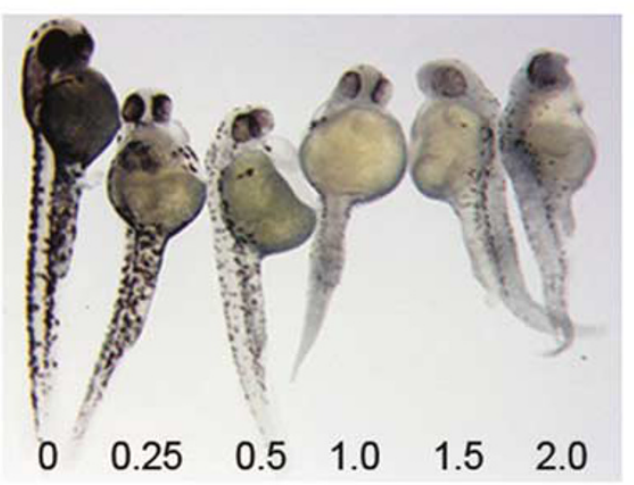

b Alco. 0

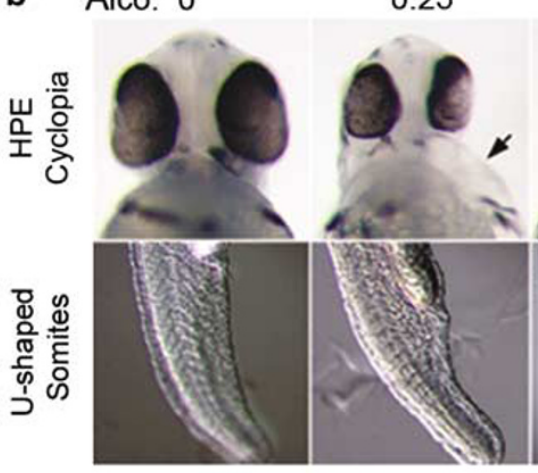

Alcohol

c
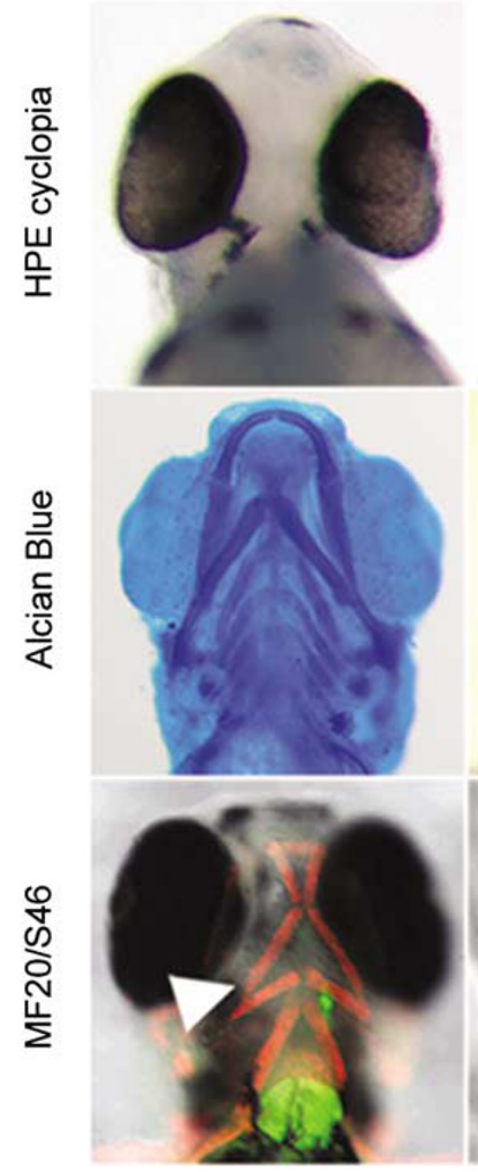
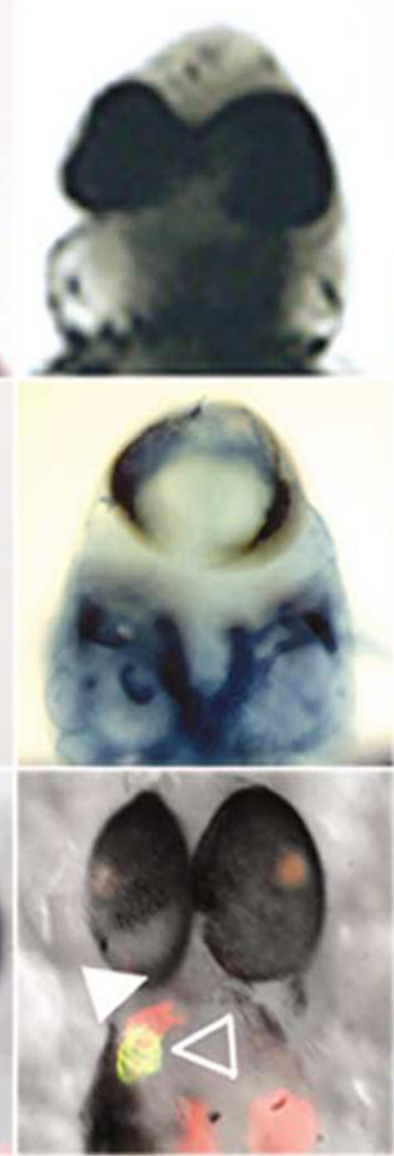

Cyc
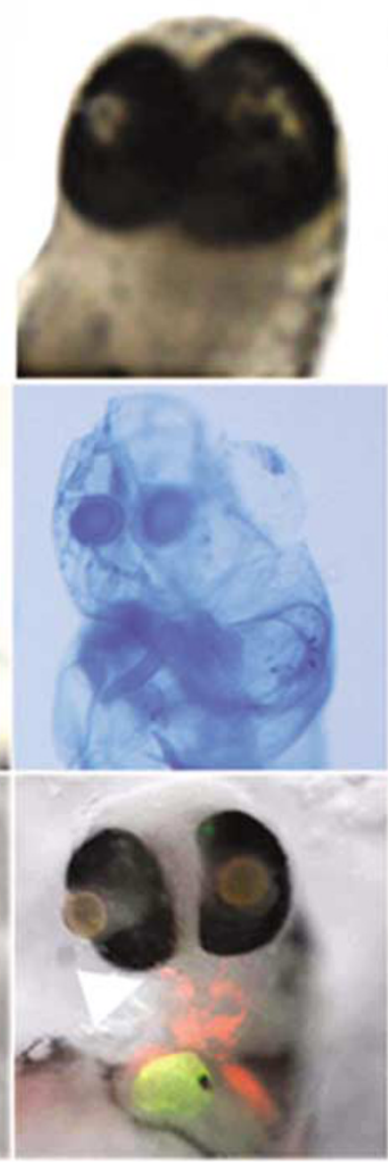

1.0 2.0

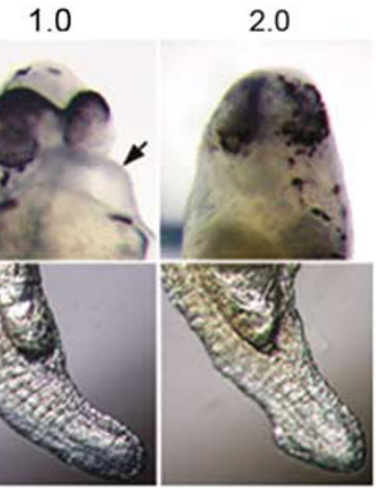

AY9944

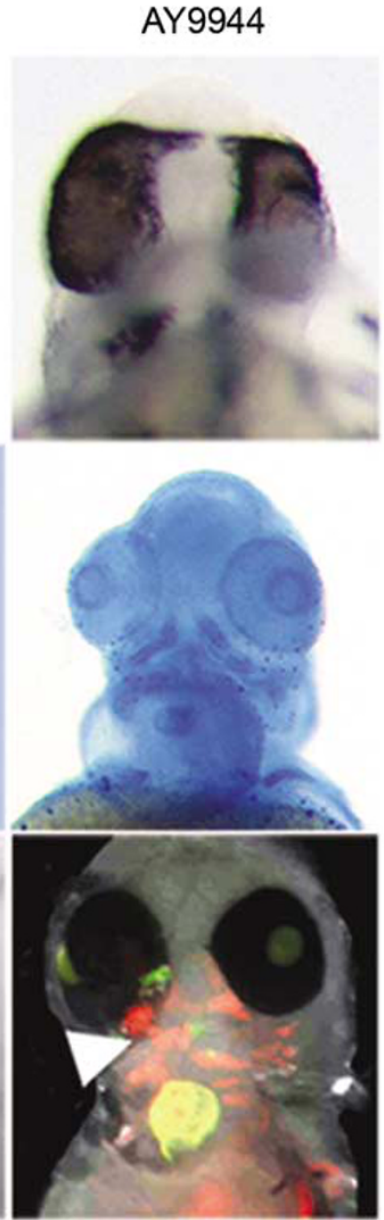

Figure 2 Fetal alcohol exposure induces a phenotype spectrum similar to that of Hh-inhibited and cholesterol deficient embryos. (a) Increasing alcohol exposures cause cranial-to-caudal defects, including generalized growth retardation in 2- day-old embryos. (b) Alcohol induces HPE, cyclopia, pericardial edema (arrow), and U-shaped somites. (c) Side-by-side comparison of 4-day-old untreated (wild-type, Wt), alcohol-exposed (2\%), cyclopamine-treated (Cyc, $10 \mu \mathrm{M})$, and AY-9944-treated (7.5 $\mu \mathrm{M})$ embryos show shared developmental malformations including HPE and cyclopia, underdeveloped of the craniofacial bones (Alcian blue)/muscles (arrowhead, MF20, red), and failure of the heart tube to loop (open arrowhead, myocardium, MF20 (red), ventricular myocardium, S46 (green/yellow)).

by RT-PCR, real-time quantitative PCR showed that Shh transcription decreased when embryos were exposed to alcohol concentrations higher than $1.0 \%$. Notably, inhibition of Shh signaling occurred despite relatively stable levels of Shh protein from whole cell lysates (cytosolic and mem- brane). However, inhibition of Shh signaling was associated with a progressive loss of Shh from the cellular membrane protein fraction isolated from FASD embryos (Figure 3d). Given that Shh must be covalently modified by cholesterol to anchor in plasma membranes, these results suggest that 
Table 1 Frequency of phenotypes in alcohol-treated embryos

\begin{tabular}{lcccccc}
\hline Alcohol (\%) & $n$ & HPE (\%) & Cyclopia (\%) & Heart edema (\%) & Arch bone defect (\%) & Defected somites shortened tail (\%) \\
\hline 0 & 147 & 0 & 0 & 0 & 0 & 0 \\
0.25 & 116 & 0.86 & 0.86 & 0.86 & 1.724 & 1.72 \\
0.5 & 123 & 1.62 & 0 & 9.76 & 1.626 & 1.63 \\
1.0 & 115 & 6.96 & 10.43 & 17.39 & 3.39 & 17.39 \\
1.5 & 92 & 28.26 & 27.17 & 28.26 & 57.70 & 40.22 \\
2.0 & 110 & 59.09 & 57.27 & 66.36 & 50.00
\end{tabular}

\footnotetext{
${ }^{a}$ Alcohol concentration in embryos medium at $\mathrm{v} / \mathrm{v}(\%)$, embryos were treated at the dome/30\% epiboly stage $(4.3 \mathrm{hpf})$ for $6 \mathrm{~h}$, phenotype were analyzed at $48 \mathrm{hpf}$ for HPE, cyclopia, and heart edema. Defective arch bones and shortened tails were analyzed at day 4 after fertilization by alcian blue staining.

${ }^{\mathrm{b}}$ Cyclopia phenotypes include full and partial cyclopia that with separate, but more closely spaced eyes.
}

cholesterol modification of Shh may be impaired in alcoholtreated embryos.

\section{Alcohol Treatment Alters Cholesterol Homeostasis and Reduced Cholesterol Ester Content}

As shown in Figure 3, alcohol exposure during the late blastula-gastrula stage causes a dose-dependent reduction in membrane-associated Shh. Given that esterification of Shh by cholesterol drives its membrane localization, these results also suggest that alcohol exposure reduces cholesterol ester formation. We tested whether alcohol exposure impairs general sterol homeostasis during gastrulation by measuring cholesterol levels in whole embryo extracts. In a dose-related fashion, alcohol exposure resulted in a decrease in the total cholesterol content of the embryos (Figure 4). This was mostly due a reduction in total cholesterol esters that paralleled the dose-dependent decreases in cholesterol-modified Shh protein and Shh signaling activity (Figure $3 a$ and $b$ ), and correlated with a dose-dependent acquisition of alcohol-induced morphologic defects: the higher the dose the more severe and extensive the defects (Figure 2a and b). Together with evidence that AY-9944 lowers cholesterol and produces similar defects (Figure 2c), our findings suggest that alcohol interrupts cholesterol homeostasis and that depleted stores of cholesterol results in impaired cholesterol modification of Shh, leading to decreased Shh signaling, which causes a FASD-like phenotype.

\section{Supplementation of Cholesterol Rescues Hh Signaling Defects Caused by Alcohol and Prevents Fetal Alcohol Induced Developmental Defects}

To confirm the importance of this potential molecular mechanism for FASD, we performed rescue experiments in alcohol-exposed embryos. Supplemental cholesterol, Gli-BSFirefly luciferase plasmid, and Renilla luciferase plasmid were co-microinjected into 1-2 cell stage embryos, which were then treated with various alcohol concentrations during gastrulation. Subsequent studies showed that Gli-BS reporter activity was preserved at all doses of alcohol exposure in cholesterol-supplemented embryos (Figure 5a). To determine whether recovery of Shh activity leads to the rescue of Hhdependent cell differentiation, we studied the slow muscle pioneer (MP) cells in somites ${ }^{42}$ using the F59 antibody which specifically identifies these progenitors. ${ }^{54}$ In untreated embryos, at $48 \mathrm{hpf}$, we observed an organized, V-shaped pattern of MP fibers with approximately 20 fibers per somite pair. Microinjection of 5\% DMSO (the cholesterol dissolving vehicle) had no adverse effect on zebrafish development. In contrast, alcohol-exposed embryos had a disorganized, diffuse pattern of MP fibers at this time. The cholesterol-supplemented, alcohol-exposed embryos had a similar number and pattern of MP fibers as the untreated, wild-type embryos (Figure 5b). Furthermore, most (94.8\%) of the cholesterolsupplemented, alcohol-exposed embryos $(n=58)$ appeared grossly normal, unlike $83.3 \%$ of the alcohol-exposed, unsupplemented embryos $(n=96)$ that had FASD-like phenotypes (Figure $5 \mathrm{c}$ ). Thus, cholesterol supplementation rescues alcohol-inhibited Shh signaling, and prevents alcohol-induced defects at the molecular, cellular, and developmental levels.

\section{DISCUSSION}

In a number of previous studies, researchers have used zebrafish to determine alcohol-related effects on development. ${ }^{55-59}$ We have extended these results by using this model to identify a novel molecular mechanism that may be responsible for alcohol's teratogenic effects, namely, alcoholinduced inhibition of the cholesterol modification of Shh, which subsequently inhibits Shh signal transduction; inhibition of this pathway appears to play the key role in the development of FASD pathogenesis.

As zebrafish lack placentas and develop ex utero, and alcohol dehydrogenases ${ }^{60,61}$ are not expressed in embryos at the time exposed to alcohol (ie from $4-10 \mathrm{hpf}$ ), Thus, the metabolites generated by oxidation of ethanol are not likely to be a major cause of the induced phenotypes. Even at very 
a

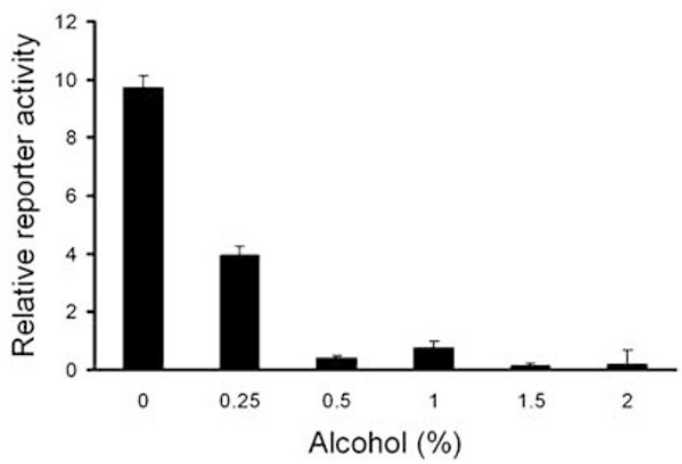

b

\begin{tabular}{|lllrrr|}
\hline \multicolumn{7}{|l|}{ Alcohol (\%) } \\
0 & 0.25 & 0.5 & 1.0 & 1.5 & 2.0 \\
\hline
\end{tabular}

Shh
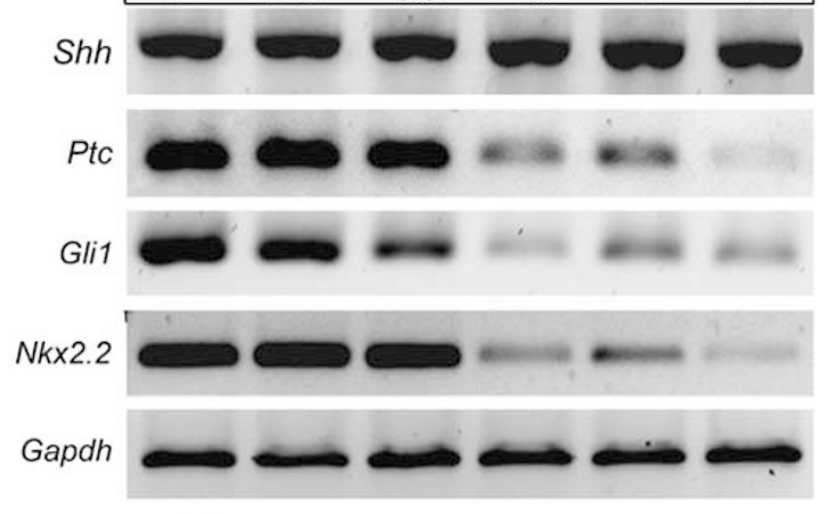

c

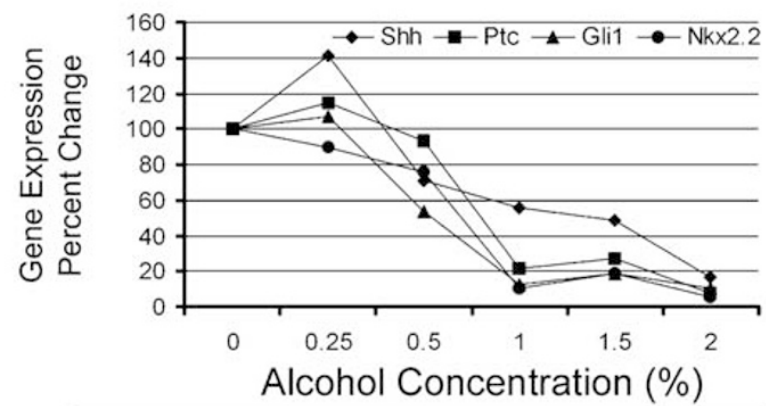

d

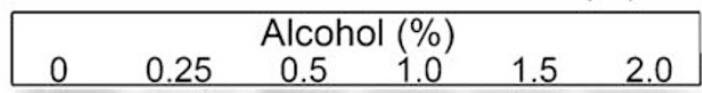

Shh

(whole ce
lysates)

Shsates

(membrane

fraction)

$\beta$-actin

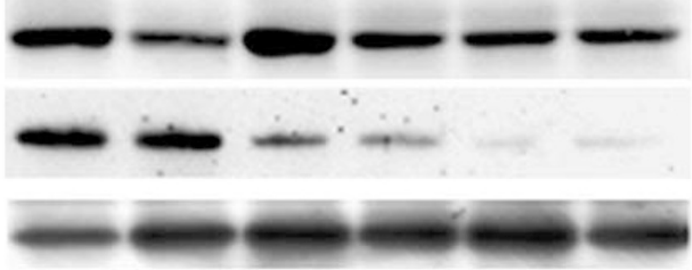

Figure 3 Alcohol exposures inhibit Hh signaling by decreasing the posttranslational cholesterol modification of Shh. (a) Dose-related reduction of a Hh-responsive Gli-luciferase activity (normalized by Renilla luciferase) in alcohol-exposed embryos. Error bars indicate 1 s.e.m. of four replicate experiments. (b) RT-PCR analysis of gene expression levels of Shh, and its target genes, Ptc, Gli1, and Nkx2.2, as well as the GAPDH housekeeping gene following alcohol exposure. (c) Semi-quantitative expression analysis of Shh and its target genes by real-time RT-PCR, data is normalized to the internal control of GAPDH. (d) Western blot of the total (cytosolic and membrane) and membrane fractions of Shh protein from alcohol-exposed embryos. $\beta$-Actin was employed as the loading control.

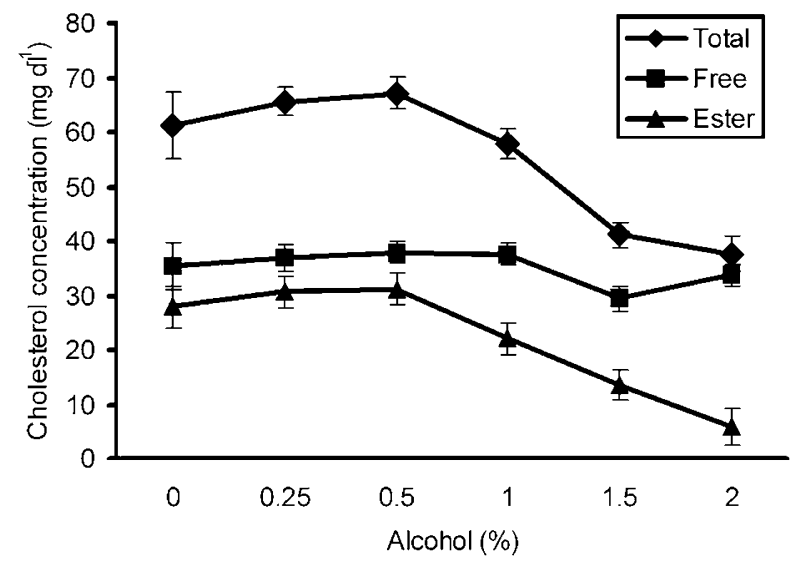

Figure 4 Alcohol exposures decrease cholesterol levels in embryos. Doserelated reduction of total cholesterol and cholesterol ester levels in alcoholexposed embryos. Error bars indicate 1 s.d.
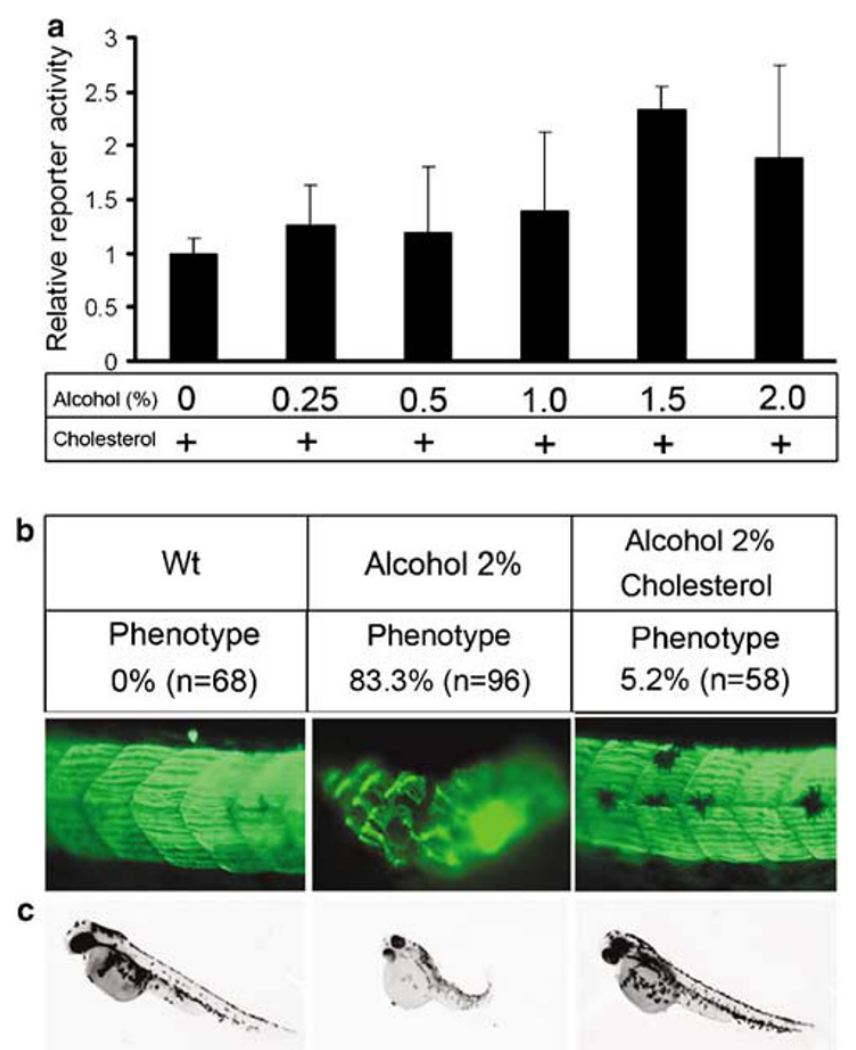

Figure 5 Cholesterol supplementation rescues alcohol-induced defects. (a) Rescue of Hh-responsive Gli-BS-luciferase reporter activity (normalized by Renilla luciferase) in alcohol-exposed embryos with cholesterol supplementation. Error bars indicate 1 s.e.m. of 11 replicate experiments. (b) Shown are untreated (Wt), alcohol-exposed (2\%), and cholesterolsupplemented (10 pg), alcohol-exposed (2\%) embryos. F59 staining of slow muscle fibers (green). (c) Lateral views of $48 \mathrm{hpf}$ zebrafish embryos demonstrate morphologic rescue in the cholesterol-supplemented, alcoholexposed embryos.

low tissue concentration, alcohol may directly causes developmental defects, instead of alcoholic metabolites from maternal resource or the embryo. 
Direct measurements determined a range of alcohol concentration from $0.71-7.4 \mathrm{mM}$ or $0.003-0.034 \mathrm{~g} \mathrm{dl}^{-1}$ in fetal tissue under our experimental conditions. These alcohol concentrations are about 5.9- to 123 -fold lower than blood alcohol levels that induce FASD in mice; ${ }^{62}$ these concentrations are also 4.2- to 153-fold lower than the alcohol concentrations that induce cell apoptosis, ${ }^{63}$ and retinoic acid deficiency ${ }^{37}$ or that have antagonistic effects on growth factors. ${ }^{36}$ Hence, relatively low concentrations of fetal tissue alcohol also can induce FASD-like defects. Blood alcohol concentrations in this range are achieved in a $55-\mathrm{kg}$ female following the consumption of one 12-ounce beer. This may explain why alcohol is the most common teratogen responsible for human congenital defects, and suggests that there is no safe level of alcohol consumption during pregnancy.

The morphological phenotypes induced by alcohol in zebrafish recapitulate the FASD defects seen in other species. For instance, similar defects have also been reported in human embryos that have FASD: $:^{23,64}$ microcephaly in $84 \%$, eye problems in $25 \%$, cardiac developmental defects in $29 \%$, various problems with truncal muscles and bones, including slack muscles in $58 \%$, swallowing/feeding problems in $20 \%$, hip deformities in $9 \%$, pidgeon chest in $30 \%$, concave chest in $7 \%$, and spinal dimple in $44 \%$. FASD patients also have craniofacial abnormalities such as facial anomalies (95\%), small teeth $(16 \%)$, cleft palate $(7 \%)$, and overall growth retardation $(98 \%)$. Thus, it seems that the ethanol-related developmental defects that we have observed in zebrafish embryos nicely parallel those observed in humans having FASD.

The evidence presented here and elsewhere ${ }^{38,65}$ consistently demonstrates that fetal alcohol exposure inhibits the transcription of Shh responsive genes. Notably, we found that total Shh protein level in the zebrafish embryonic tissues was not significantly changed by any of the tested alcohol exposures. However, the treated embryos exhibited FASD-like phenotypes and impaired $\mathrm{Hh}$ signal transduction, suggesting that defective Shh signaling is the key factor in the morphological defects induced by alcohol. Furthermore, we have now shown that the defect in Shh signal transduction is due to disruption of the post-translational cholesterol modification of Shh. This findings help to explain why over-expression of Shh mRNA alone does not consistently rescue alcohol-induced morphologic defects or the decrease in expression of Shh responsive genes. ${ }^{38,65}$

Here, we observed that supplementing a simple chemical, cholesterol rescues the alcohol-inhibited Hh signal transduction and prevents embryos from developing FASD-like morphologic defects. Recently, sterols were shown to directly activate the Shh signaling pathway through Smo. Cholesterol, or certain oxyterols, serve as Smo agonists. ${ }^{66}$ This evidence suggests that cholesterol may also directly stimulate Smo to initiate Shh signal transduction, bypassing the pathway and rescuing alcohol-induced Shh-dependent development. It will be interesting to determine whether the same cholesterol supplementation strategy that rescues alcohol-exposed embryos might protect embryos from tetratogenicity caused by other environmental factors that induce cholesterol-related defects in Shh signaling.

The mature Shh peptide is doubly lipid-modified, having a cholesterol moiety at its $\mathrm{C}$ terminus, ${ }^{4}$ and a palmitate attachment at Cys-24 of the $\mathrm{N}$ terminus. ${ }^{67}$ The N-terminal lipid is required for inducing the differentiation of ventral forebrain neurons. ${ }^{68}$ In contrast, in the absence of the Nlipid, the C-terminal lipid-containing Shh is sufficient to induce mouse digit duplication. ${ }^{69}$ Mouse mutants have been created in which Shh lacks cholesterol modification, lacks palmitoylation, or lacks both types of lipid modification. Functional analysis of these mutants clearly demonstrated that both types of lipid modification are essential for regulating the range and shape of the Shh morphogen gradient during early development. ${ }^{70,71}$ For future direction, it remains to be determined whether the alcohol-induced defect in cholesterol modification influences $\mathrm{N}$-terminal palmitoylation, or Shh cellular trafficking in lipid rafts, or affects the binding affinity of Shh to Ptc, or even the gradient shape and content of Shh.

Overall, we have shown that a simple post-translational modification defect of a key morphogen results in a complex congenital disease. This new insight into the molecular basis of FASD has far-reaching implications, and suggests novel prenatal interventions that might prevent FASD developmental defects.

Supplementary Information accompanies the paper on the Laboratory Investigation website (http://www.laboratoryinvestigation.org)

\section{ACKNOWLEDGEMENTS}

We thank Dr Caron MG, Dr Modrich PL, Dr Jhaveri R, and Dr Morris DP for criticisms and discussion of the manuscript. This work was supported by $\mathrm{NIH}$ AA016144 (YXL) and AHA 0430250N (YXL), NIH AA010154 (AMD), R01 DK053792 (AMD), R01 AA012059 (AMD), T32 DK007568 (HY) and T32 DK007713 (JKS).

1. Mann RK, Beachy PA. Novel lipid modifications of secreted protein signals. Annu Rev Biochem 2004;73:891-923.

2. Hammerschmidt M, Brook A, McMahon AP. The world according to hedgehog. Trends Genet 1997;13:14-21.

3. Nybakken K, Perrimon N. Heparan sulfate proteoglycan modulation of developmental signaling in Drosophila. Biochim Biophys Acta 2002;1573:280-291.

4. Porter JA, Young KE, Beachy PA. Cholesterol modification of hedgehog signaling proteins in animal development. Science 1996;274:255-259.

5. Feng J, White B, Tyurina OV, et al. Synergistic and antagonistic roles of the Sonic hedgehog $\mathrm{N}$ - and C-terminal lipids. Development 2004;131:4357-4370.

6. Stone DM, Hynes M, Armanini M, et al. The tumour-suppressor gene patched encodes a candidate receptor for Sonic hedgehog. Nature 1996;384:129-134.

7. Ding $Q$, Fukami $S$, Meng $X$, et al. Mouse suppressor of fused is a negative regulator of sonic hedgehog signaling and alters the subcellular distribution of Gli1. Curr Biol 1999;9:1119-1122.

8. Concordet JP, Lewis KE, Moore JW, et al. Spatial regulation of a zebrafish patched homologue reflects the roles of sonic hedgehog and 
protein kinase A in neural tube and somite patterning. Development 1996;122:2835-2846.

9. Dai $P$, Akimaru $H$, Tanaka $Y$, et al. Sonic Hedgehog-induced activation of the Gli1 promoter is mediated by GLI3. J Biol Chem 1999;274: 8143-8152.

10. Barth KA, Wilson SW. Expression of zebrafish nk2.2 is influenced by sonic hedgehog/vertebrate hedgehog-1 and demarcates a zone of neuronal differentiation in the embryonic forebrain. Development 1995;121:1755-1768.

11. Echelard Y, Epstein DJ, St-Jacques B, et al. Sonic hedgehog, a member of a family of putative signaling molecules, is implicated in the regulation of CNS polarity. Cell 1993;75:1417-1430.

12. Roelink H, Augsburger A, Heemskerk J, et al. Floor plate and motor neuron induction by vhh-1, a vertebrate homolog of hedgehog expressed by the notochord. Cell 1994;76:761-775.

13. Riddle $\mathrm{RD}$, Johnson $\mathrm{RL}$, Laufer $\mathrm{E}$, et al. Sonic hedgehog mediates the polarizing activity of the ZPA. Cell 1993;75:1401-1416.

14. Johnson RL, Laufer E, Riddle RD, et al. Ectopic expression of Sonic hedgehog alters dorsal-ventral patterning of somites. Cell 1994;79:1165-1173.

15. Wallis D, Muenke M. Mutations in holoprosencephaly. Hum Mutat 2000;16:99-108.

16. Ronen GM, Andrews WL. Holoprosencephaly as a possible embryonic alcohol effect. Am J Med Genet 1991;40:151-154.

17. Siebert JR, Astley SJ, Clarren SK. Holoprosencephaly in a fetal macaque (Macaca nemestrina) following weekly exposure to ethanol. Teratology 1991;44:29-36.

18. Coulter CL, Leech RW, Schaefer GB, et al. Midline cerebral dysgenesis, dysfunction of the hypothalamic-pituitary axis, and fetal alcohol effects. Arch Neurol 1993;50:771-775.

19. Croen LA, Shaw GM, Lammer EJ. Risk factors for cytogenetically normal holoprosencephaly in California: a population-based case-control study. Am J Med Genet 2000;90:320-325.

20. Belloni E, Muenke M, Roessler E, et al. Identification of Sonic hedgehog as a candidate gene responsible for holoprosencephaly. Nat Genet 1996;14:353-356.

21. Ericson J, Morton S, Kawakami A, et al. Two critical periods of Sonic Hedgehog signaling required for the specification of motor neuron identity. Cell 1996;87:661-673.

22. Roebuck TM, Simmons RW, Richardson C, et al. Neuromuscular responses to disturbance of balance in children with prenatal exposure to alcohol. Alcohol Clin Exp Res 1998;22:1992-1997.

23. Loser $\mathrm{H}$, Bierstedt $\mathrm{T}$, Blum A. Fetal alcohol syndrome in adulthood. A long-term study. Dtsch Med Wochenschr 1999;124:412-418.

24. Kyllerman M, Aronson M, Sabel KG, et al. Children of alcoholic mothers. Growth and motor performance compared to matched controls. Acta Paediatr Scand 1985;74:20-26.

25. Connor PD, Sampson PD, Streissguth AP, et al. Effects of prenatal alcohol exposure on fine motor coordination and balance: A study of two adult samples. Neuropsychologia 2006;44:744-751.

26. Chudley AE, Conry J, Cook JL, et al. Fetal alcohol spectrum disorder: Canadian guidelines for diagnosis. Can Med Assoc J 2005;172:S1-S21.

27. Wada $\mathrm{N}$, Javidan $\mathrm{Y}$, Nelson $\mathrm{S}$, et al. Hedgehog signaling is required for cranial neural crest morphogenesis and chondrogenesis at the midline in the zebrafish skull. Development 2005;132:3977-3988.

28. Mann RK, Beachy PA. Cholesterol modification of proteins. Biochim Biophys Acta 2000;1529:188-202.

29. Zeng X, Goetz JA, Suber LM, et al. A freely diffusible form of Sonic hedgehog mediates long-range signalling. Nature 2001;411:716-720.

30. Cooper MK, Porter JA, Young KE, et al. Teratogen-mediated inhibition of target tissue response to Shh signaling. Science 1998;280: 1603-1607.

31. Leiber B. Alcohol-currently the most significant agent causing malformations. Severe multiple abnormalities in children of alcoholics are increasing at an alarming rate. A warning from DOFONOS. Med Monatsschr Pharm 1978;1:25-26.

32. Jones KL, Smith DW. Recognition of the fetal alcohol syndrome in early infancy. Lancet 1973;2:999-1001.

33. Olney JW, Tenkova T, Dikranian K, et al. Ethanol-induced caspase-3 activation in the in vivo developing mouse brain. Neurobiol Dis 2002;9:205-219

34. Charness ME, Safran RM, Perides G. Ethanol inhibits neural cell-cell adhesion. J Biol Chem 1994;269:9304-9309.
35. Kotch LE, Chen SY, Sulik KK. Ethanol-induced teratogenesis: free radical damage as a possible mechanism. Teratology 1995;52: 128-136.

36. Henderson Gl, Baskin GS, Horbach J, et al. Arrest of epidermal growth factor-dependent growth in fetal hepatocytes after ethanol exposure. J Clin Invest 1989;84:1287-1294.

37. Deltour L, Ang HL, Duester G. Ethanol inhibition of retinoic acid synthesis as a potential mechanism for fetal alcohol syndrome. FASEB J 1996;10:1050-1057.

38. Blader $\mathrm{P}$, Strahle U. Ethanol impairs migration of the prechordal plate in the zebrafish embryo. Dev Biol 1998;201:185-201.

39. Yelin R, Schyr RB, Kot $\mathrm{H}$, et al. Ethanol exposure affects gene expression in the embryonic organizer and reduces retinoic acid levels. Dev Biol 2005;279:193-204.

40. Solnica-Krezel L. Conserved patterns of cell movements during vertebrate gastrulation. Curr Biol 2005;15:R213-R228.

41. Tanaka Y, Okada Y, Hirokawa N. FGF-induced vesicular release of Sonic hedgehog and retinoic acid in leftward nodal flow is critical for leftright determination. Nature 2005;435:172-177.

42. Barresi MJ, Stickney HL, Devoto SH. The zebrafish slow-muscle-omitted gene product is required for Hedgehog signal transduction and the development of slow muscle identity. Development 2000; 127:2189-2199.

43. Kelley RL, Roessler E, Hennekam RC, et al. Holoprosencephaly in RSH/ Smith-Lemli-Opitz syndrome: does abnormal cholesterol metabolism affect the function of Sonic Hedgehog? Am J Med Genet 1996;66: 478-484.

44. Dehart DB, Lanoue L, Tint GS, et al. Pathogenesis of malformations in a rodent model for Smith-Lemli-Opitz syndrome. Am J Med Genet 1997;68:328-337.

45. Cooper MK, Wassif CA, Krakowiak PA, et al. A defective response to Hedgehog signaling in disorders of cholesterol biosynthesis. Nat Genet 2003;33:508-513.

46. Polo MP, de Bravo MG, de Alaniz MJ. Effect of ethanol on cell growth and cholesterol metabolism in cultured Hep G2 cells. Biochem Cell Biol 2003;81:379-386.

47. Axelson M, Mork B, Sjovall J. Ethanol has an acute effect on bile acid biosynthesis in man. FEBS Lett 1991;281:155-159.

48. Buchmann MS, Kvittingen EA, Nazer $\mathrm{H}$, et al. Lack of 3 beta-hydroxydelta 5-C27-steroid dehydrogenase/isomerase in fibroblasts from a child with urinary excretion of 3 beta-hydroxy-delta 5-bile acids. A new inborn error of metabolism. J Clin Invest 1990;86:2034-2037.

49. Gigliozzi A, Romeo R, Fraioli F, et al. Effect of S-adenosyl-L-methionine and dilinoleoylphosphatidylcholine on liver lipid composition and ethanol hepatotoxicity in isolated perfused rat liver. Dig Dis Sci 1998:43:2211-2222.

50. Carrasco MP, Segovia JL, Marco C. Incorporation of exogenous precursors into neutral lipids and phospholipids in rat hepatocytes: effect of ethanol in vitro. Biochem Pharmacol 1998;56:1639-1644.

51. Wilbanks AM, Fralish GB, Kirby ML, et al. Beta-arrestin 2 regulates zebrafish development through the hedgehog signaling pathway. Science 2004;306:2264-2267.

52. Sicklick JK, Li YX, Choi SS, et al. Role for hedgehog signaling in hepatic stellate cell activation and viability. Lab Invest 2005;85: 1368-1380.

53. Sasaki H, Hui C, Nakafuku M, et al. A binding site for Gli proteins is essential for HNF-3beta floor plate enhancer activity in transgenics and can respond to Shh in vitro. Development 1997;124:1313-1322.

54. Crow MT, Stockdale FE. Myosin expression and specialization among the earliest muscle fibers of the developing avian limb. Dev Biol 1986;113:238-254.

55. Bilotta J, Barnett JA, Hancock L, et al. Ethanol exposure alters zebrafish development: a novel model of fetal alcohol syndrome. Neurotoxicol Teratol 2004;26:737-743.

56. Bilotta J, Saszik S, Givin CM, et al. Effects of embryonic exposure to ethanol on zebrafish visual function. Neurotoxicol Teratol 2002;24:759-766.

57. Arenzana FJ, Carvan III MJ, Aijon J, et al. Teratogenic effects of ethanol exposure on zebrafish visual system development. Neurotoxicol Teratol 2006;28:342-348.

58. Carvan III MJ, Loucks E, Weber DN, et al. Ethanol effects on the developing zebrafish: neurobehavior and skeletal morphogenesis. Neurotoxicol Teratol 2004;26:757-768. 
59. Reimers MJ, Flockton AR, Tanguay RL. Ethanol- and acetaldehydemediated developmental toxicity in zebrafish. Neurotoxicol Teratol 2004:26:769-781.

60. Dasmahapatra AK, Doucet HL, Bhattacharyya C, et al. Developmental expression of alcohol dehydrogenase (ADH3) in zebrafish (Danio rerio) Biochem Biophys Res Commun 2001;286:1082-1086.

61. Reimers MJ, Hahn ME, Tanguay RL. Two zebrafish alcohol dehydrogenases share common ancestry with mammalian class I, II, IV and $\mathrm{V}$ alcohol dehydrogenase genes but have distinct functional characteristics. J Biol Chem 2004;279:38303-38312.

62. Sulik KK, Johnston MC, Webb MA. Fetal alcohol syndrome: embryogenesis in a mouse model. Science 1981;214:936-938.

63. Ikonomidou C, Bittigau P, Ishimaru MJ, et al. Ethanol-induced apoptotic neurodegeneration and fetal alcohol syndrome. Science 2000;287:1056-1060.

64. Von Knappen B, Thater H, Loeser H. Alkoholschaeden bei Kindern. Ratgeber zur Alkoholembryopathie. Lambertus Verlag Freiburg: Germany, 1996.

65. Ahlgren SC, Thakur V, Bronner-Fraser M. Sonic hedgehog rescues cranial neural crest from cell death induced by ethanol exposure. Proc Natl Acad Sci USA 2002;99:10476-10481.
66. Corcoran RB, Scott MP. Oxysterols stimulate Sonic hedgehog signal transduction and proliferation of medulloblastoma cells. Proc Nat Acad Sci USA 2006;103:8408-8413.

67. Pepinsky RB, Zeng C, Wen D, et al. Identification of a palmitic acid-modified form of human Sonic hedgehog. J Biol Chem 1998;273:14037-14045.

68. Kohtz JD, Lee HY, Gaiano N, et al. N-terminal fatty-acylation of sonic hedgehog enhances the induction of rodent ventral forebrain neurons. Development 2001;128:2351-2363.

69. Lee JD, Treisman JE. Sightless has homology to transmembrane acyltransferases and is required to generate active Hedgehog protein. Curr Biol 2001;11:1147-1152.

70. Lewis PM, Dunn MP, McMahon JA, et al. Cholesterol modification of sonic hedgehog is required for long-range signaling activity and effective modulation of signaling by Ptc1. Cell 2001;105: 599-612.

71. Chen MH, Li YJ, Kawakami T, et al. Palmitoylation is required for the production of a soluble multimeric Hedgehog protein complex and long-range signaling in vertebrates. Genes Dev 2004;18: 641-659. 